\title{
Immunoassay for LMPI in nasopharyngeal tissue based on surface-enhanced Raman scattering
}

This article was published in the following Dove Press journal:

International Journal of Nanomedicine

29 December 201I

Number of times this article has been viewed

\author{
Yanping Chen ${ }^{*}$ \\ Xiongwei Zheng ${ }^{*}$ \\ Gang Chen "* \\ Chen $\mathrm{He}^{\prime}$ \\ Weifeng Zhu' \\ Shangyuan Feng ${ }^{2}$ \\ Gangqin $\mathrm{Xi}^{2}$ \\ Rong Chen ${ }^{2}$ \\ Fenghua $\operatorname{Lan}^{3}$ \\ Haishan Zeng ${ }^{4}$ \\ 'Pathology Department of Fujian \\ Provincial Tumor Hospital, Teaching \\ Hospital of Fujian Medical University, \\ ${ }^{2}$ Key Laboratory of OptoElectronic \\ Science and Technology for Medicine, \\ Ministry of Education, Fujian Normal \\ University, ${ }^{3}$ Research Center for \\ Molecular Diagnosis of Genetic \\ Diseases, Fuzhou General Hospital, \\ Clinical College of Fujian Medical \\ University, Fuzhou, Fujian, People's \\ Republic of China; ${ }^{4}$ maging Unit, \\ Integrative Oncology Department, \\ British Columbia Cancer Agency \\ Research Centre, Vancouver, Canada \\ *These authors contributed equally \\ to this work
}

Correspondence: Fenghua Lan Research Center for Molecular Diagnosis of Genetic Diseases, Fuzhou General Hospital, I56 Xihuanbei Road, Fuzhou, Fujian 350025 ,

People's Republic of China

Tel +86 59| 8372 II 05

Fax +86 59। 8372 ।I05

Email fhlan009@qq.com
Background: Previous studies have shown that Epstein-Barr virus (EBV)-encoded latent membrane protein 1 (LMP1) is closely associated with the occurrence and development of nasopharyngeal carcinoma, and can be used as a tumor marker in screening for the disease. Here we report a new methodology based on highly specific and sensitive surface-enhanced Raman scattering (SERS) technology to detect LMP1 in nasopharyngeal tissue sections directly with no need of tedious procedures as with conventional immunohistochemistry methods.

Methods: LMP1-functionalized 4-mercaptobenzoic acid (4-MBA)-labeled Au/Ag core-shell bimetallic nanoparticles were prepared first and then applied for analyzing LMP1 in formalinfixed paraffin-embedded nasopharyngeal tissue sections obtained from 34 cancer patients and 20 healthy controls. SERS spectra were acquired from a $25 \times 25$ spot square area on each tissue section and used to generate SERS images.

Results: Data from SERS spectra and images show that this new SERS-based immunoassay detected LMP1 in formalin-fixed paraffin-embedded nasopharyngeal tissue sections with high sensitivity and specificity. The results from the new LMP1-SERS probe method are superior to those of conventional immunohistochemistry staining for LMP1, and in excellent agreement with those of in situ hybridization for EBV-encoded small RNA (EBER).

Conclusion: This new SERS technique has the potential to be developed into a new clinical tool for detection and differential diagnosis of nasopharyngeal carcinoma as well as for predicting metastasis and immune-targeted treatment of nasopharyngeal carcinoma.

Keywords: surface-enhanced Raman scattering, immunoassay, LMP1, nasopharyngeal carcinoma, in situ hybridization, immunohistochemistry

\section{Introduction}

Nasopharyngeal carcinoma is a strongly Epstein-Barr virus (EBV)-associated malignancy that is particularly prevalent in southeast China, Hong Kong, Singapore, Taiwan, and Malaysia, where the incidence is high, with 20-30 cases per 100,000. ${ }^{1}$ Recent studies have clearly demonstrated that EBV-encoded latent membrane protein 1 (LMP1) functions as a classical oncogene in rodent-fibroblast transformation assays and is essential for EBV-induced B cell transformation in vitro. ${ }^{2}$ In addition, LMP1 has pleiotropic effects when it is expressed in cells, resulting in blockade of differentiation in epithelial cells, upregulation of DNA methyltransferase activity, upregulation of antiapoptotic proteins $\left(\mathrm{BCL}_{2}\right)$, and so on. ${ }^{3}$ Therefore, LMP1 can be used as a tumor marker to screen for nasopharyngeal carcinoma and for predicting metastasis and immune-targeted treatment of the disease. ${ }^{4,5}$ 
Currently, immunohistochemistry is the most widely used method to detect LMP1 expression in tissue sections. However, conventional immunohistochemistry is timeconsuming and has relatively low detection sensitivity and accuracy. Although EBV is associated with more than $90 \%$ of nasopharyngeal carcinoma, the reported detectable expression rates of LMP1 determined by immunohistochemistry techniques were from $50 \%$ to $80 \% .^{5-7}$ Therefore, there is incentive to develop new techniques that overcome such limitations to provide an accurate, fast, and sensitive method for LMP1 detection.

With the help of metal nanostructures, surface-enhanced Raman scattering (SERS) can provide $10^{6}-10^{14}$-fold of enhancement in Raman signal intensity, which is sufficient even for single-molecule detection. ${ }^{8}$ Therefore, SERS-based immunoassay based on specific interactions between an antigen and a complementary antibody has emerged as a novel and promising technique in biomedicine..$^{9-12}$ In recent years, this technology has become a versatile tool for the study of biomolecules such as DNA and RNA, ${ }^{13,14}$ pathogens, ${ }^{15}$ and tumor markers, ${ }^{11,16-19}$ as well as circulating tumor cells in human peripheral blood, ${ }^{10}$ even in vivo tumor target detection. ${ }^{20}$ However, there are few reports about SERS-based immunoassay for protein expression in paraffin tissue sections. ${ }^{16-18,21}$

Herein, we developed a method that uses Au/Ag core-shell nanoparticles as SERS substrates and 4-mercaptobenzoic acid (4-MBA) as Raman reporters for LMP1 detection in formalin-fixed paraffin-embedded nasopharyngeal tissue sections and demonstrated high sensitivity and specificity. To the best of our knowledge, this is the first report on LMP1conjugated 4-MBA-labeled $\mathrm{Au} / \mathrm{Ag}$ core-shell nanoparticles combined with SERS imaging for detection of LMP1, which may be developed into a new clinical tool for providing diagnostic and prognostic information on patients with nasopharyngeal carcinoma.

\section{Materials and methods Reagents}

Mouse anti-LMP1 monoclonal antibody was purchased from BioTeke Company, Beijing, China. Bovine serum albumin (BSA) was purchased from Genview, Germany. 4-MBA trisodium citrate, glutaraldehyde, $\mathrm{HAuCl}_{4} \cdot 4 \mathrm{H}_{2} \mathrm{O}$, and silver nitrate $\left(\mathrm{AgNO}_{3}\right)$ were obtained from Beijing Dingguo Changsheng Biotechnology Co Ltd. All the chemicals used were of analytical grade. Borate buffer solution $(2 \mathrm{mM}$, $\mathrm{pH} 9.0)$, citrate buffer (0.01 M, pH 6.0), and phosphate buffer solution (0.01 M, pH 7.2-7.4) were purchased from BioTeke Company. All solutions required in the experiments were prepared using deionized water with a conductivity of $18 \mathrm{M} \Omega \cdot \mathrm{cm}$ at $25^{\circ} \mathrm{C}$.

\section{Preparation of LMPI-conjugated SERS nanoparticles}

Briefly, bimetallic Au/Ag core-shell nanoparticles were prepared by the seed-growth method as described elsewhere. ${ }^{22,23}$ Then, $4 \mu \mathrm{L}\left(1.0 \times 10^{-3} \mathrm{~mol} / \mathrm{L}\right) 4-\mathrm{MBA}$ as Raman-active molecules were added to $1.0 \mathrm{~mL}$ of colloids $\left(0.5 \times 10^{-4} \mathrm{~mol} / \mathrm{L}\right)$. Under continuous stirring for 12 hours, the probe-labeled colloids were purified by centrifugation at $10,000 \mathrm{rpm}$ for 10 minutes and resuspended in $1.0 \mathrm{~mL}$ borate buffer solution. Next, $5 \mu \mathrm{L}$ of $2.0 \mathrm{mg} / \mathrm{mL}$ antiLMP1 monoclonal antibodies were added to $1.0 \mathrm{~mL}$ of 4-MBA-labeled colloids. After reacting for one hour at room temperature, the probe-labeled immuno- $\mathrm{Au} / \mathrm{Ag}$ colloids were purified by centrifugation and resuspended in $1.0 \mathrm{~mL}$ of borate buffer solution. The concentration of anti-LMP1 monoclonal antibody in the final solution was $1.0 \times 10^{-2} \mathrm{mg} / \mathrm{mL}$. This amount of anti-LMP1 is $50 \%$ over the minimum amount for coating the unmodified portion of the colloid surface. Finally, $10 \mu \mathrm{L}$ bovine serum albumin (BSA) $(2 \%, \mathrm{~m} / \mathrm{m})$ were added to the probe-labeled immuno-Au/Ag colloids (as shown in Figure 1A). The mixture was incubated for one hour at room temperature, then centrifuged and resuspended in $1.0 \mathrm{~mL}$ borate buffer solution. LMP1-SERS probes are stable for several months when stored at $4^{\circ} \mathrm{C}$.

\section{Tissue sample collection}

In this study, tissue samples were collected from 34 patients with primary and cervical lymph node metastases from nasopharyngeal carcinoma and 20 healthy volunteers. All patients were from Fujian Provincial Tumor Hospital and had similar ethnic and socioeconomic backgrounds.

\section{A}
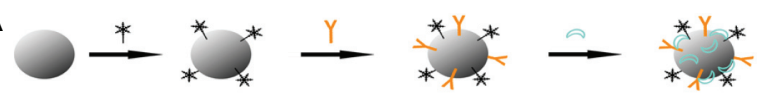

B
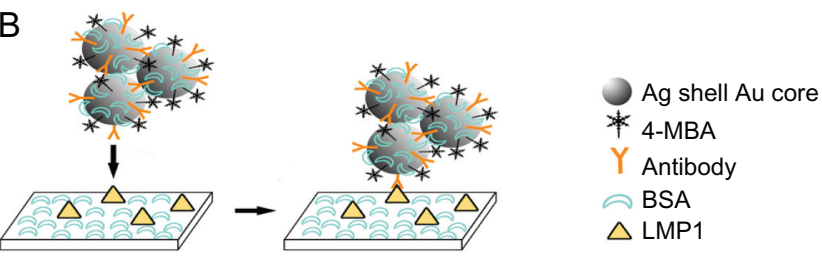

Figure I Preparation processes of the latent membrane protein I surfaceenhanced Raman scattering probe-based immunoassay. (A) Schematic illustration of the preparation processes of 4-mercaptobenzoic acid-labeled $\mathrm{Au} / \mathrm{Ag}$ core-shell nanoparticles; (B) schematic procedure of a direct binding assay.

Abbreviations: 4-MBA, 4-mercaptobenzoic acid; BSA, bovine serum albumin; LMPI, latent membrane protein I. 
Diagnoses were carried out by two experienced pathologists according to the 2005 World Health Organization (WHO) histological criteria and classification. There were 33 cases of non-keratinizing undifferentiated carcinomas (WHO type III) and one case of non-keratinizing differentiated carcinoma (WHO type II). The control samples were obtained from normal nasopharyngeal tissues (for more detailed information, see Table S1). All the study subjects provided written consent and ethics permission was obtained for the study.

\section{LMPI-SERS staining and SERS measurement}

After antigen retrieval with citrate buffer $(\mathrm{pH} \mathrm{5.5,2} \mathrm{mM})$ in a steamer, formalin-fixed paraffin-embedded tissue sections (thickness about $4 \mu \mathrm{m}$ ) were blocked with $3 \%$ bovine serum albumin/phosphate buffer solution for 20 minutes at room temperature to provide general protein blocking and incubated with LMP1-SERS probes in a humidity chamber for 2 hours at room temperature (as shown in Figure 1B). The slides were washed twice with phosphate buffer solution and air-dried at room temperature before measurement.

A Renishaw Raman microscope (InVia system, UK) was used for the collection of SERS spectra. A $785 \mathrm{~nm}$ near-infrared diode laser was used as the excitation source. The spectra were collected using a Leica $20 \times$ objective, and the detection of Raman signal was carried out with a Peltier-cooled CCD camera. Raman spectra in the range of 400-1800 $\mathrm{cm}^{-1}$ were acquired with an integration time of one second. SERS images were performed using the global Raman imaging technology by raster scanning the laser beam across the imaging field. The integration time for each spectrum was one second and the spacing between adjacent scanning spots was $2 \mu \mathrm{m}$. For generating a SERS map $(25 \times 25$ spots $)$ were scanned over a small square area on a tissue section. The SERS spectra were processed with the software package Wire 2.0 (Renishaw).

\section{Data processing and analysis}

The least-squares approach was applied to analyze qualitatively the LMP1-SERS signal from every tissue sample. This method allows deconvolution of the LMP1SERS probe signal from autofluorescence and other background signals. From the derived LMP1-SERS probe spectra, the peak intensity at $1596 \mathrm{~cm}^{-1}$ for each of the $(25 \times 25$ spots $)$ was calculated to form a SERS image. The Raman peak intensity level at each pixel is coded by pseudo colors. Black represents the lowest intensity, while white represents the highest. Each pixel on the SERS image was then classified as LMP-1-positive or LMP-1-negative using a single fixed threshold value of 40,000 au. We have quantified the accuracy of staining for repeated measurements within different sites on multiple tissue samples, and we routinely find accuracies greater than $90 \%$.

\section{Immunohistochemistry staining of LMPI}

Immunohistochemical analysis for LMP1 expression on formalin-fixed paraffin-embedded tissue sections were performed using an Elivision plus Polyer horseradish peroxidase mouse immunohistochemistry kit (Maixin Bio, Fuzhou, China). The slides were incubated with antiLMP1 monoclonal antibody according to the manufacturer's protocol.

\section{In situ hybridization for EBER}

In situ hybridization studies for EBV-encoded small RNA (EBER) were performed using an EBER detection kit (Triplex International Biosciences, Fuzhou, China) according to the manufacturer's protocol.

\section{Results \\ Characterization of LMPI-SERS nanoparticles}

The structure of $\mathrm{Au} / \mathrm{Ag}$ core-shell nanoparticles with good monodispersity can be seen clearly on the transmission electron microscopic (TEM) image (Figure 2A left panel). The average size of the core-shell particles is about 26-28 nm. The Au/Ag core-shell colloids display a more uniform distribution of size than do silver colloids and no rod-shaped particles are observed, while rod-shaped particles are observed in the TEM images for silver colloids (see Figure S1). As shown in Figure 2A (right panel), appropriate 4-MBA can induce slight colloid aggregation and form significant SERS-active nanoclusters, leading to a great enhancement in Raman signals.

From the ultraviolet-visible absorption spectra (Figure 2B), we can see that the strong absorption peak of pure $\mathrm{Au} / \mathrm{Ag}$ core-shell nanoparticles (black curve) is at $399 \mathrm{~nm}$. After addition of a certain amount of 4-MBA, the intensity of the $\mathrm{Au} / \mathrm{Ag}$ core-shell nanoparticle absorption band reduced markedly and the absorption peak red-shifted to $404 \mathrm{~nm}$ (red curve), suggesting that the monodispersed core-shell nanoparticles aggregated slightly. ${ }^{24}$ However, no new plasma resonance absorption peak appeared in the longer red/near infrared wavelength region, indicating that $4 \mu \mathrm{L}$ of $4-\mathrm{MBA}$ did not induce heavy colloid aggregation and severe sedimentation. ${ }^{24}$ 
A
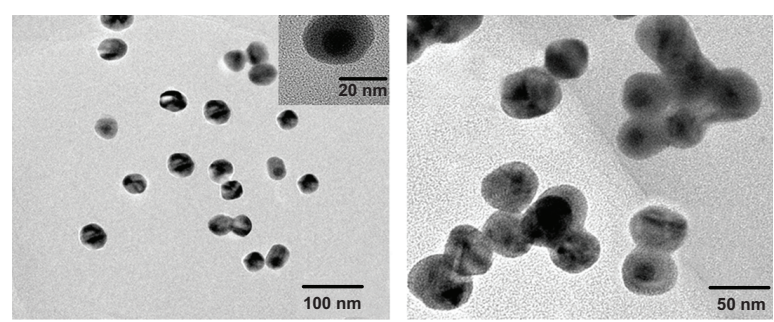

B

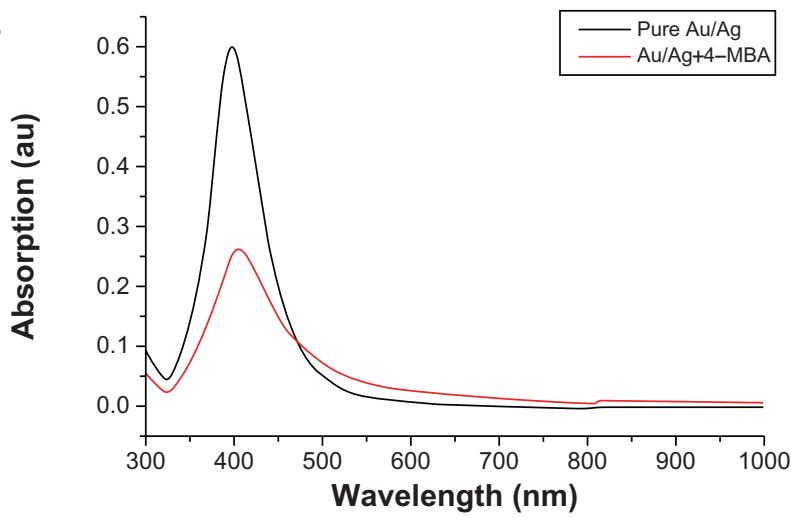

C

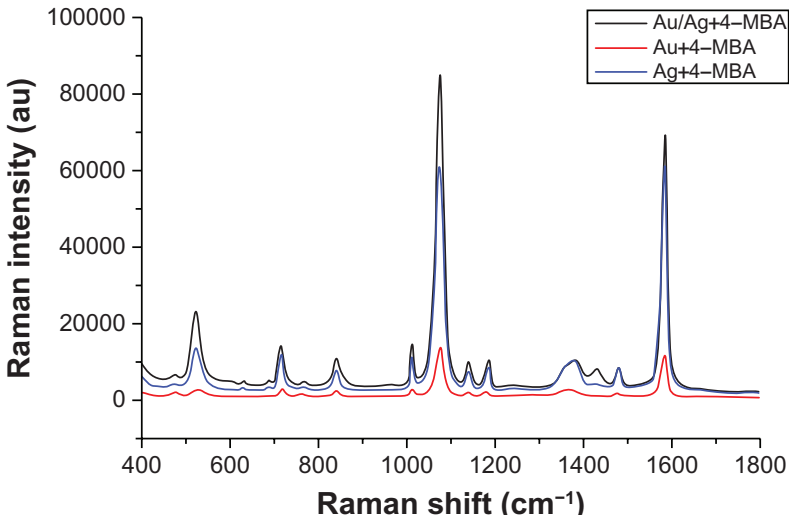

Figure 2 (A) Transmission electron microscopic images of $\mathrm{Au} / \mathrm{Ag}$ core-shell nanoparticle colloids (left) and composite nanoparticles with 4-mercaptobenzoic acid (right); (B) ultraviolet-visible absorption spectra of pure $\mathrm{Au} / \mathrm{Ag}$ core-shell nanoparticles colloid (black curve) and $\mathrm{Au} / \mathrm{Ag}$ core-shell nanoparticles with 4-mercaptobenzoic acid (red curve); (C) surface-enhanced Raman scattering spectra of 4-mercaptobenzoic acid adsorbed onto gold nanoparticles, silver nanoparticles, and $\mathrm{Au} / \mathrm{Ag}$ core-shell nanoparticles, respectively.

SERS spectra were measured to validate the SERS activity of different substrates. As shown in Figure 2C, gold nanoparticles show a very weak Raman spectrum of 4-MBA (red curve), indicating that gold nanoparticles are less optimal SERS-active substrates. However, silver nanoparticles exhibit a very strong Raman signal, suggesting that silver nanoparticles are good SERS-active substrates (blue curve). The bimetallic $\mathrm{Au} / \mathrm{Ag}$ core-shell nanoparticles display a much stronger and better resolved spectrum for 4-MBA (black curve) than that from the monometallic gold and silver nanoparticles, demonstrating that the $\mathrm{Au} / \mathrm{Ag}$ coreshell nanoparticle is a better SERS-active substrate than pure silver and gold nanoparticles.
Figure $3 \mathrm{~A}$ is the Raman spectrum for pure $\mathrm{Au} / \mathrm{Ag}$ core-shell colloids. The signal is very noisy and no reliable Raman peaks are seen, indicating that there is no interference signal for 4-MBA. Figure 3B shows the native Raman spectrum of solid 4-MBA, which has two obvious and unique Raman bands at $1099 \mathrm{~cm}^{-1}$ and $1596 \mathrm{~cm}^{-1}$, but the signal is weak. After blending 4-MBA and $\mathrm{Au} / \mathrm{Ag}$ core-shell colloids together, the Raman peaks at $1099 \mathrm{~cm}^{-1}$ and $1596 \mathrm{~cm}^{-1}$ shift to $1079 \mathrm{~cm}^{-1}$ and $1586 \mathrm{~cm}^{-1}$, and Raman signals were enhanced significantly via both electromagnetic and chemical enhancement mechanisms (as shown in Figure 3C). There are no reliable Raman peaks in the SERS spectrum of $\mathrm{Au} / \mathrm{Ag}$ core-shell colloids with the LMP1 antibody (Figure 3D). However, after subsequent addition of 4-MBA, the unique SERS signals of 4-MBA become dominant (Figure 3E) and the Raman signals were enhanced significantly. It is obvious that this new functionalized LMP1 and 4-MBA-labeled

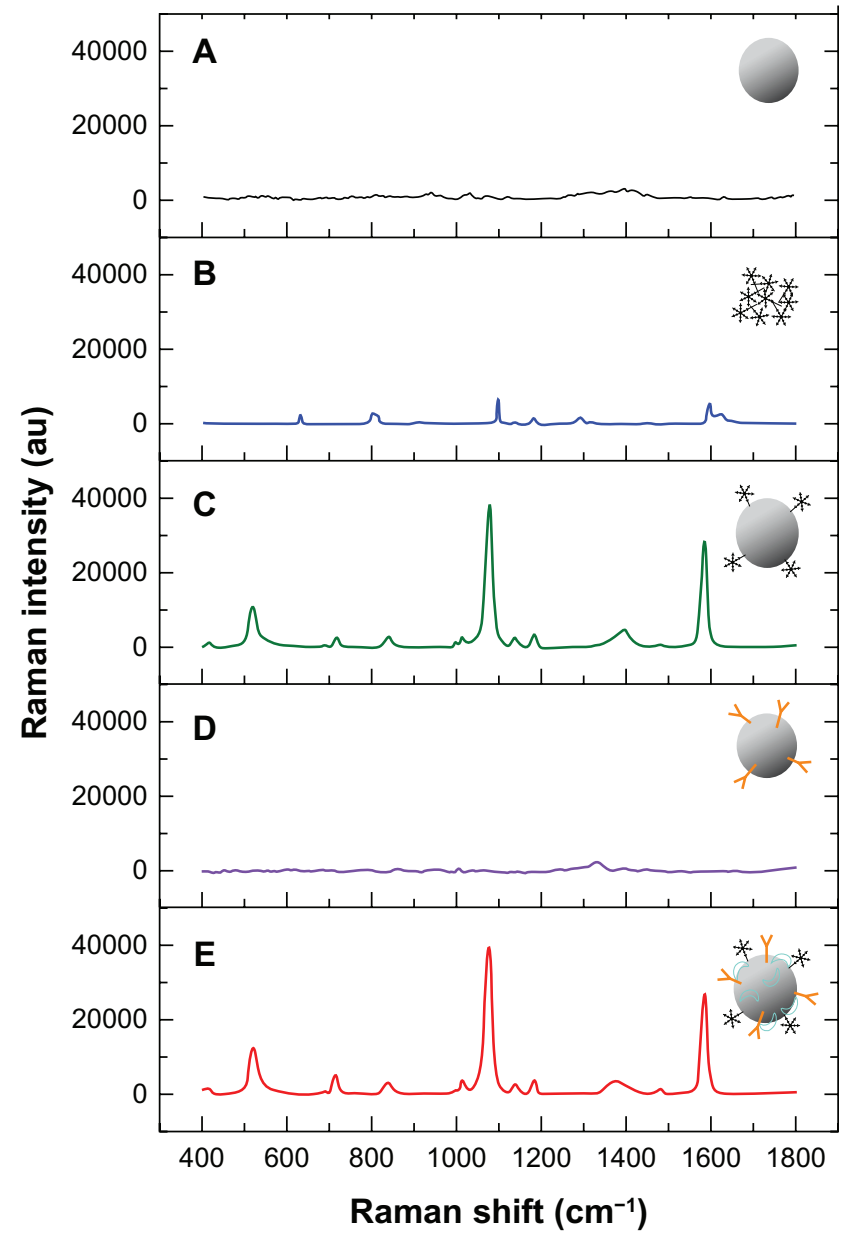

Figure 3 Raman spectra of Au/Ag core-shell colloids (A) and solid 4-mercaptobenzoic acid (B), as well as the surface-enhanced Raman scattering spectra of 4-mercaptobenzoic acid $\mathrm{Au} / \mathrm{Ag}$ nanoparticles (C), latent membrane protein I $\mathrm{Au} / \mathrm{Ag}$ nanoparticles (D), and 4-mercaptobenzoic acid latent membrane protein I $\mathrm{Au} / \mathrm{Ag}$ nanoparticles (E), respectively. 
$\mathrm{Au} / \mathrm{Ag}$ core-shell nanoparticles have high stability, strong SERS activity, and good immune specificity which can be used for highly effective SERS-based immunoassay.

\section{Detection sensitivity and specificity of LMPI - SERS probe}

SERS enhances Raman signal intensity by $10^{6}-10^{14}$, and is capable of achieving single-molecule detection sensitivity. Therefore, the main challenge of this assay was to minimize nonspecific binding of nanoparticles. In order to assess the performance of this new LMP1-SERS probe, cancerous nasopharyngeal tissue which overexpressed LMP1 was used to test the targeting specificity and sensitivity of LMP1-SERS nanoparticles, while normal nasopharyngeal tissue which did not express LMP1 was used as a control.

The bright field images of a region from normal and cancerous nasopharyngeal tissues were recorded prior to each SERS mapping scan (Figure 4A and B). The white boxes in Figure 4A and $\mathrm{B}$ indicate the imaging fields of normal nasopharyngeal epithelial tissue and nasopharyngeal cancer cells, respectively. The in situ detection of LMP1 in normal and cancerous nasopharyngeal tissue sections by Raman spectroscopy is shown in Figure 4C. The typical SERS spectrum collected from cancer tissue displays a strong Raman signal, which is attributed to specific binding of the LMP1 antibody, whereas only negligible SERS signals are observed in normal tissue, which is likely due to nonspecific adsorption of the LMP1-SERS probe. Furthermore, to verify the specificity of the LMP1-SERS probe for cancer tissue, another negative control experiment was carried out to detect the binding of nontargeted bovine serum albumin-SERS probes to cancer tissue. There were no reliable Raman peaks observed in cancer tissue. The spectrum at the bottom in Figure $4 \mathrm{C}$ is the strong background autofluorescence signal of glass incubated with the LMP1-SERS probe, which is characterized by a broad emission at $1365 \mathrm{~cm}^{-1}$. We see no interference Raman peaks in the spectral range of interest where the SERS probe Raman signals appear.

In addition, SERS imaging, which is a powerful tool for determining the distribution of biochemical substances in biological specimens, was applied to create mappings of LMP1 expression patterns in normal and cancerous nasopharyngeal tissue sections, where black represents the lowest intensity and white represents the highest. As can be seen in Figure 4D, normal nasopharyngeal epithelial tissue shows as black almost everywhere except for a few dark red spots, which are most likely due to nonspecific binding of the LMP1 antibody, indicating that there is no
A

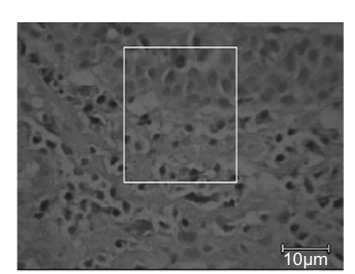

B

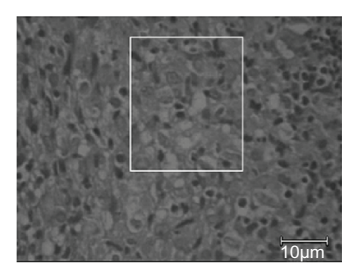

C

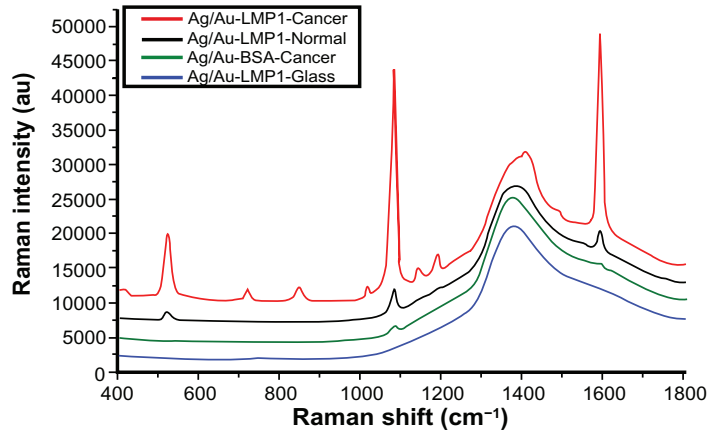

D

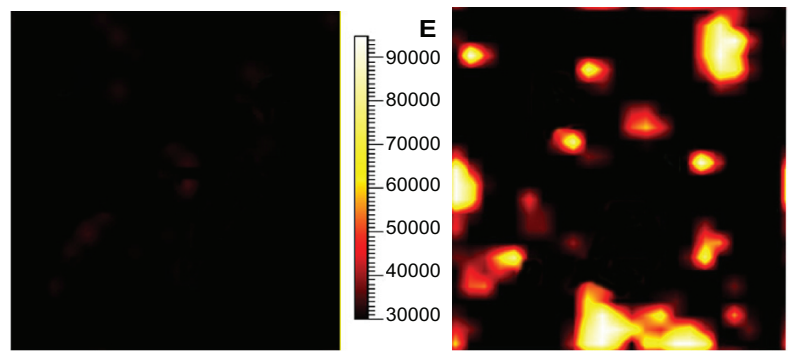

Figure 4 (A) and (B) Bright field images of a region from examples of normal and cancerous nasopharyngeal tissue sections, respectively; (C) are the representative spectra obtained from latent membrane protein I surface-enhanced Raman scattering probes in cancerous nasopharyngeal tissue (red), normal nasopharyngeal tissue (blue) and glass (black), as well as nontargeted surface-enhanced Raman scattering probes (bovine serum albumin surface-enhanced Raman scattering probes) in cancerous nasopharyngeal tissue (green); (D) and (E) demonstrate surface-enhanced Raman scattering images of example of normal tissue and cancerous tissue, respectively. The surface-enhanced Raman scattering images were acquired by scanning the laser beam to cover the white boxes in (A) and (B), respectively.

expression of LMP1. In Figure 4E, cancer cells appear as yellow and white, indicating high expression of LMP1 in nasopharyngeal cancer cells. This result is in good agreement with the LMP1 expression patterns expected in normal and cancer tissues.

\section{Application of LMPI-SERS probes on nasopharyngeal tissue sections}

To evaluate the reliability and feasibility of the proposed immunoassay for clinical diagnosis, a series of samples from 34 patients with nasopharyngeal carcinoma and 20 healthy subjects were analyzed simultaneously with the new LMP1SERS probe method (as shown in Figures S3 and S4), conventional immunohistochemistry, and in situ hybridization (as shown in Figure S2). Using the conventional immunohistochemistry technique, we observed that LMP1 was positive in 22 of the 34 patients with nasopharyngeal carcinoma 
(64.7\%) and negative in all 20 healthy controls. However, all patients with nasopharyngeal carcinoma were EBER-in situ hybridization (ISH)-positive (100\%) and all healthy subjects were negative $(100 \%)$ by EBER-ISH, which is recognized as a preferred method for detection of EBV infection with high specificity and sensitivity in present clinical practice. ${ }^{25}$ Finally, with LMP1-SERS probe staining, LMP1 was positive in 33 of the 34 patients with nasopharyngeal carcinoma (97.1\%) and negative in all 20 healthy subjects (100\%). It is obvious that the SERS results were superior to those of conventional immunohistochemistry staining for LMP1 and consistent with those of in situ hybridization for EBER. This suggests that the new LMP1-SERS probe method has potential for development into a clinical tool for detecting LMP1 expression in paraffin tissue sections.

\section{Discussion}

\section{SERS-active substrate}

According to our experiments, Au/Ag core-shell nanoparticles produced much stronger and better resolved SERS spectra than those from monometallic gold or silver nanoparticles. The Au/Ag core-shell nanoparticle is a better SERS-active substrate than the pure silver and gold nanoparticle. This result is consistent with the literature. ${ }^{22,23}$ This phenomenon may be explained in terms of three aspects. First, the ultraviolet-visible absorbance spectra indicates that the optical properties of the composite nanoparticles are dominated by the silver shell. Most studies have found that silver is more powerful than gold as a SERS-active substrate. ${ }^{22,23}$ Thus, it is not surprising that $\mathrm{Au} / \mathrm{Ag}$ core-shell colloids exhibit stronger Raman enhancement than gold nanoparticles. In the TEM observation, it is interesting to find that the $\mathrm{Au} / \mathrm{Ag}$ core-shell colloids display more uniform distribution of size and higher homogeneity of the silver surface than silver colloids. Thus, the Au/Ag coreshell colloids can produce greater enhancement of Raman signals of 4-MBA, and the enhancement is more consistent due to the homogeneity of the surface structure. Furthermore, the electronic ligand effect in bimetallic nanoparticles and localized electric field enhancement can also induce a great enhancement effect. Because the Fermi level for gold is lower than that for silver, the gold atoms in the core can have a strong electronic effect on the surface silver atoms by charge transfer, causing the surface silver atoms adjacent to the gold core to become more active than the silver atoms in monometallic silver nanoparticles. ${ }^{26}$ Therefore, as a SERS-active substrate, $\mathrm{Au} / \mathrm{Ag}$ core-shell nanoparticles can enhance Raman signals of 4-MBA more effectively than gold nanoparticles and silver nanoparticles.

\section{Optimum quantity of 4-MBA}

The Raman label concentration is critical for obtaining probelabeled immune- $\mathrm{Au} / \mathrm{Ag}$ core-shell colloids of appropriate size and stability. ${ }^{27}$ If the concentration is too low, there would be no significant cluster formation and the resulting colloids would have very low Raman scattering intensity. In contrast, when the label concentration is too high, there would be rapid aggregation which could even lead to instantaneous agglomeration. ${ }^{18,28}$ The degree of aggregation depends greatly on the amount of Raman label added. ${ }^{24}$ Because of the strong adsorbability of the thiol group on SERS probe molecules such as 4-MBA, the double electronic layer around the colloid particle surface coated by citrate would be destroyed and lead to instability and aggregation of colloid particles. Consequently, agglomeration would reduce the accuracy of SERS measurement.

From TEM observation, we found that the Au/Ag coreshell nanoparticles had good monodispersity. However, slight aggregation to severe sedimentation can occur by adding different amounts of 4-MBA. The red shift of the absorption peak corresponds to an increase in particle size, which not only demonstrated successful binding of 4-MBA to the nanoparticles but also reflected the amounts of 4-MBA added. With the increasing amounts of 4-MBA, the absorption peak of $\mathrm{Au} / \mathrm{Ag}$ core-shell nanoparticles shifted to the red side gradually. Simultaneously, the Raman reporter-labeled aggregates indicate strong SERS activities. If the concentration of 4-MBA was too high, this would lead to instability of the colloid particles and affect the SERS measurements. According to our experiments, the SERS probe generated by adding $4 \mu \mathrm{L} 4-\mathrm{MBA}$ into $1.0 \mathrm{~mL} \mathrm{Au} / \mathrm{Ag}$ core-shell colloids exhibited better stability and gratifying SERS activity. This result is in good agreement with Ji et al. ${ }^{23}$ The quantity of 4-MBA only occupies a portion of the composite nanoparticle surface and there is still some space left for antibody. The optimum quantity of 4-MBA ensures high stability of the SERS probes, strong SERS activity, and good immune specificity. Such a SERS probe can accomplish a highly sensitive SERS-labeled immunoassay.

\section{Advantages of SERS immunoassay}

Compared with conventional immunohistochemistry, our new SERS method has unique advantages, ie, a singlestep staining procedure, which may dramatically decrease the time involved in a traditional immunohistochemistry experiment, such as incubation with secondary antibodies, enzymatic color development time, and endogenous biotin blocking, as well as ultrasensitive detection. SERS can 
also provide a $10^{6}-10^{14}$-fold enhancement in Raman signal intensity that is sufficient for single-molecule level detection without amplification of the secondary antibody. Raman bands are much narrower than fluorescence bands, resulting in a larger pool of available and nonoverlapping Raman probes, which offers the potential to develop multiplexed detection capabilities in a single tissue sample..$^{16,17,19,29}$ In addition, the SERS immunoassay combined with the SERS imaging technique can rapidly, accurately, and conveniently analyze the spatial distribution of proteins in tissue sections, providing useful diagnostic and prognostic information for cancer patients.

\section{Conclusion}

We have developed a highly specific and sensitive methodology using 4-MBA labeled Au/Ag core-shell nanoparticles combined with a SERS imaging technique for rapid analysis of LMP1 expression in nasopharyngeal cancer tissue. The reliability and feasibility of the new method was validated using tissue samples from 34 patients with nasopharyngeal carcinoma and 20 healthy subjects. The results were superior to those of conventional immunohistochemistry staining for LMP1 and consistent with those for in situ hybridization for EBER. Our study has demonstrated the feasibility and promise of developing this new technology into a clinical tool for differential diagnosis of nasopharyngeal carcinoma, as well as monitoring for recurrence and progression of patients with the disease. Furthermore, this method has the potential to be extended for detecting multiple antigens in a tissue sample simultaneously.

\section{Acknowledgments}

We thank Long-feng Ke for excellent technical assistance, and Jing Wang, Yun Yu, Zu-fang Huang and Xiao-qian Lin for their support with this project and helpful comments on the manuscript. The study was supported by the National Natural Science Foundation of China (11104030, 61178090, 81101110) and the Canadian Institutes of Health Research International Scientific Exchange Program.

\section{Disclosure}

The authors report no conflicts of interest in this work.

\section{References}

1. Young LS, Rickinson AB. Epstein-Barr virus: 40 years on. Nat Rev Cancer. 2004;4:757-768.

2. Wang D, Liebowitz D, Kieff E. An EBV membrane protein expressed in immortalized lymphocytes transforms established rodent cells. Cell. 1985;43:831-840.

3. Li HP, Chang YS. Epstein-Barr virus latent membrane protein 1: structure and functions. J Biomed Sci. 2003;10:490-504.
4. Tao D, Xu J, Chen R, et al. Detection of EBV-LMP1 antibodies in sera and clinical significance in the patients with nasopharyngeal carcinoma. Lin Chung Er Bi Yan Hou Tou Jing Wai Ke Za Zhi. 2011;25:401-405. Chinese.

5. Zhao Y, Wang Y, Zeng S, et al. LMP1 expression is positively associated with metastasis of nasopharyngeal carcinoma: evidence from a meta-analysis. J Clin Pathol. October 29, 2011. [Epub ahead of print.]

6. Li J, Zhang XS, Xie D, et al. Expression of immune-related molecules in primary EBV-positive Chinese nasopharyngeal carcinoma: associated with latent membrane protein 1 (LMP1) expression. Cancer Biol Ther. 2007;6:1997-2004.

7. Bar-Sela G, Kuten A, Minkov I, et al. Prevalence and relevance of EBV latency in nasopharyngeal carcinoma in Israel. J Clin Pathol. 2004;57: 290-293.

8. Kneipp J, Kneipp H, Kneipp K. SERS-a single-molecule and nanoscale tool for bioanalytics. Chem Soc Rev. 2008;37:1052-1060.

9. Xu S, Ji X, Xu W, et al. Surface-enhanced Raman scattering studies on immunoassay. J Biomed Opt. 2005;10:031112.

10. Wang X, Qian X, Beitler JJ, et al. Detection of circulating tumor cells in human peripheral blood using surface-enhanced Raman scattering nanoparticles. Cancer Res. 2011;71:1526-1532.

11. Tang D, Su B, Tang J, Ren J, Chen G. Nanoparticle-based sandwich electrochemical immunoassay for carbohydrate antigen 125 with signal enhancement using enzyme-coated nanometer-sized enzyme-doped silica beads. Anal Chem. 2010;82:1527-1534.

12. Ravindran R, Khan IH, Krishnan VV, et al. Validation of multiplex microbead immunoassay for simultaneous serodetection of multiple infectious agents in laboratory mouse. J Immunol Methods. 2010; 363:51-59.

13. Cao YWC, Jin R, Mirkin CA. Nanoparticles with Raman spectroscopic fingerprints for DNA and RNA detection. Science. 2002;297: $1536-1540$.

14. Liang Y, Gong JL, Huang Y, et al. Biocompatible core-shell nanoparticle-based surface-enhanced Raman scattering probes for detection of DNA related to HIV gene using silica-coated magnetic nanoparticles as separation tools. Talanta. 2007;72:443-449.

15. Lin C, Yang Y, Chen Y, Yang T, Chang H. A new protein A assay based on Raman reporter labeled immunogold nanoparticles. Biosens Bioelectron. 2008;24:178-183.

16. Lutz B, Dentinger C, Nguyen L, et al. Spectral analysis of multiplex Raman probe signatures. ACS Nano. 2008;2:2306-2314.

17. Lutz B, Dentinger C, Sun L, et al. Raman nanoparticle probes for antibody-based protein detection in tissues. J Histochem Cytochem. 2008;56:371-379.

18. Sun L, Sung K, Dentinger C, et al. Composite organic-inorganic nanoparticles as Raman labels for tissue analysis. Nano Lett. 2007;7: 351-356.

19. Yoon K, Seo H, Hwang H, et al. Bioanalytical application of SERS immunoassay for detection of prostate-specific antigen. Bull Korean Chem Soc. 2010;31:1215-1218.

20. Qian X, Peng XH, Ansari DO, et al. In vivo tumor targeting and spectroscopic detection with surface-enhanced Raman nanoparticle tags. Nat Biotechnol. 2007;26:83-90.

21. Schlücker S, Küstner B, Punge A, Bonfig R, Marx A, Ströbel P. Immuno-Raman microspectroscopy: In situ detection of antigens in tissue specimens by surface-enhanced Raman scattering. J Raman Spectrosc. 2006;37:719-721.

22. Yang Y, Shi J, Kawamura G, Nogami M. Preparation of Au-Ag, Ag-Au core-shell bimetallic nanoparticles for surface-enhanced Raman scattering. Scripta Mater. 2008;58:862-865.

23. Ji X, Xu S, Wang L, et al. Immunoassay using the probe-labeled Au/Ag core-shell nanoparticles based on surface-enhanced Raman scattering. Colloids Surf Physicochem Eng Aspects. 2005;257:171-175.

24. Song C, Wang Z, Zhang R, Yang J, Tan X, Cui Y. Highly sensitive immunoassay based on Raman reporter-labeled immuno-Au aggregates and SERSactive immune substrate. Biosens Bioelectron. 2009;25: 826-831. 
25. Mirzamani N, Salehian P, Farhadi M. Detection of EBV and HPV in nasopharyngeal carcinoma by in situ hybridization. Exp Mol Pathol. 2006;81:231-234.

26. Kumar GVP, Shruthi S, Vibha B, Reddy BAA, Kundu TK, Narayana C. Hot spots in Ag core-Au shell nanoparticles potent for surface-enhanced Raman scattering studies of biomolecules. J Phys Chem C. 2007;111:4388-4392.

27. Xu S, Ji X, Xu W, et al. Immunoassay using probe-labelling immunogold nanoparticles with silver staining enhancement via surface-enhanced Raman scattering. Analyst. 2004;129:63-68.
28. Su X, Zhang J, Sun L, et al. Composite organic-inorganic nanoparticles (COINs) with chemically encoded optical signatures. Nano Lett. 2005; $5: 49-54$.

29. Chon H, Lim C, Ha S, et al. On-chip immunoassay using surfaceenhanced Raman scattering of hollow gold nanospheres. Anal Chem. 2010;82:5290-5295.

30. Frens G. Controlled nucleation for the regulation of the particle size in monodisperse gold solutions. Nat Phys Sci. 1973;241:20-22. 


\section{Supplementary information Materials and methods}

Preparation of gold core/Ag shell colloid solutions

Briefly, the gold nanoparticles were prepared according to Frens, ${ }^{30}$ then $5 \mathrm{~mL}$ of gold colloids $\left(1 \times 10^{-3} \mathrm{~mol} / \mathrm{L}\right)$ were diluted up to $100 \mathrm{~mL}$, heated to boiling point under stirring, and $1.0 \mathrm{~mL}$ of $1 \%$ trisodium citrate solution was then added. Under continuous boiling, $0.5 \mathrm{~mL}$ of $\mathrm{AgNO}_{3}\left(1.0 \times 10^{-2}\right.$ $\mathrm{mol} / \mathrm{L}$ ) was added every 5 minutes (ten times in total) to produce the desired final bimetallic colloids.

\section{Preparation of tissue sections}

Biopsy specimens from patients and healthy controls were fixed in $10 \%$ neutral buffered formalin and embedded in paraffin blocks. Four pieces of $4 \mu \mathrm{m}$ thick sections were cut from each block using a microtome. One was stained with hematoxylin-eosin as the reference section for pathological verification. The other three were used for surface-enhanced Raman scattering probe staining, immunohistochemistry staining, and in situ hybridization, respectively. All tissue sections were baked at $60^{\circ} \mathrm{C}$ for 2 hours and were then deparaffinized with xylene, rehydrated through graded ethanol (three changes in $100 \%$ ethanol for 2 minutes each, two changes in $95 \%$ ethanol for 2 minutes each, and one change of $70 \%$ ethanol for 1 minute) for further experiments.

\section{Results}
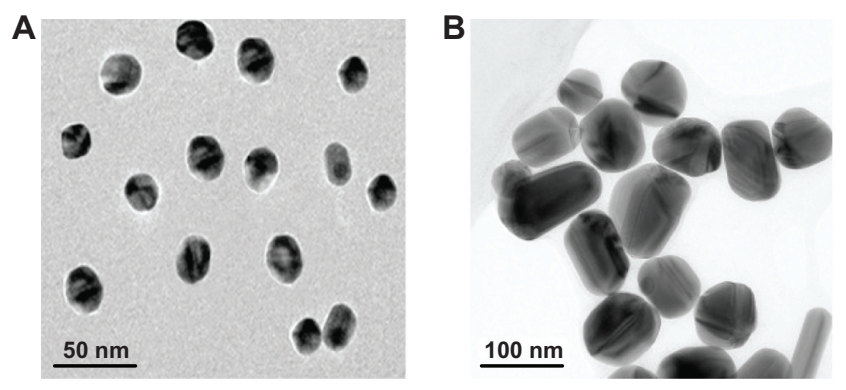

Figure SI Transmission electron microscopic images of the $\mathrm{Au} / \mathrm{Ag}$ core-shell nanoparticles (A) and pure silver nanoparticles (B).
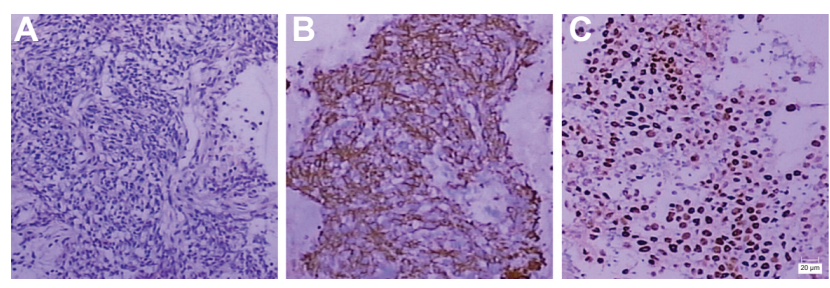

Figure S2 A biopsy specimen obtained from a patient with undifferentiated nonkeratinizing nasopharyngeal carcinoma: (A) hematoxylin-eosin staining $(\times 100)$; (B) cytoplasmic staining of latent membrane protein I by immunohistochemistry (stained in brown) $(\times 100)(\mathbf{C})$ in situ hybridization for Epstein-Barr virus-encoded small RNA (positive, stained in brown, $\times 100$ ).

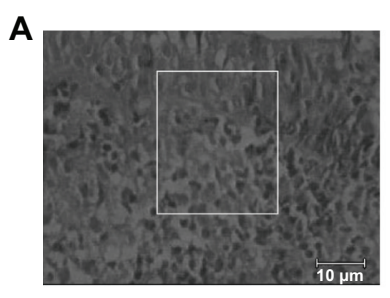

B
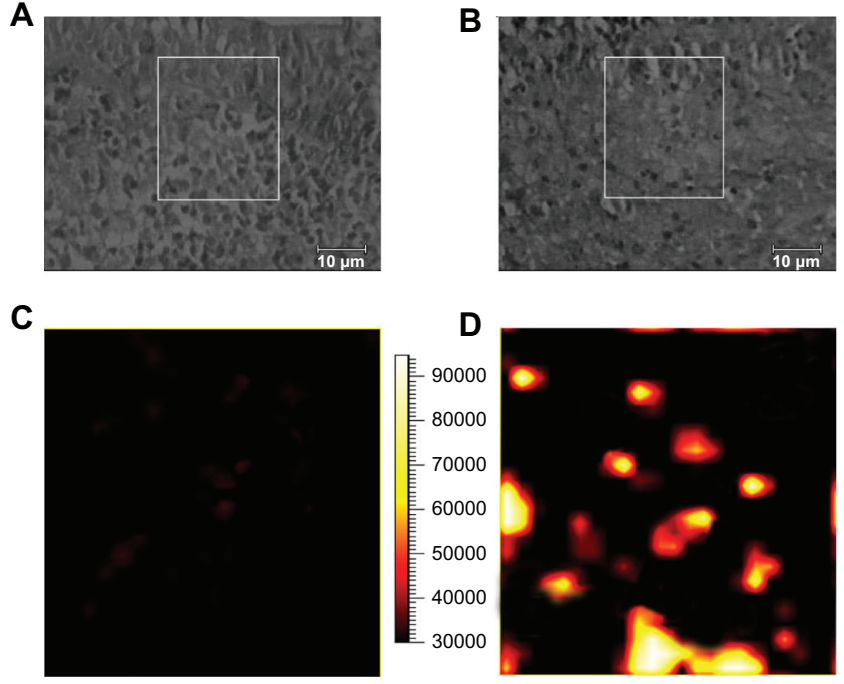

Figure S3 (A) and (B) Bright field images of a region from normal and cancerous nasopharyngeal tissue sections, respectively; (C) and (D) demonstrate surfaceenhanced Raman scattering images of normal tissue and cancerous tissue, respectively. The surface-enhanced Raman scattering images were acquired by scanning the laser beam to cover the white boxes in $(\mathbf{A})$ and $(\mathbf{B})$, respectively.

A

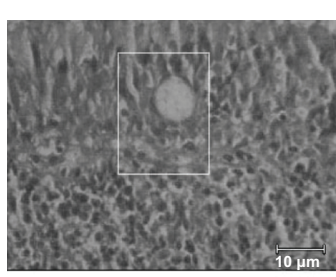

B

C
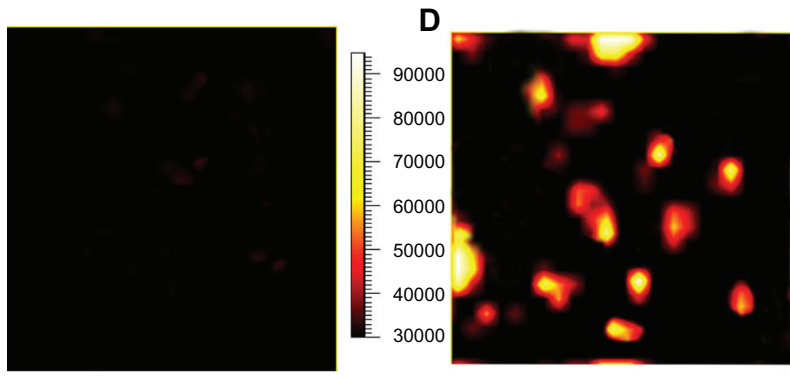

Figure S4 (A) and (B) Bright field images of a region from normal and cancerous nasopharyngeal tissue sections, respectively; (C) and (D) demonstrate surfaceenhanced Raman scattering images of normal tissue and cancerous tissue, respectively. The surface-enhanced Raman scattering images were acquired by scanning the laser beam to cover the white boxes in $(\mathbf{A})$ and $(\mathbf{B})$ respectively. 
Table SI Summary of clinical data from patients and results of LMPI surface-enhanced Raman scattering and LMPI immunohistochemistry staining, as well as EBER-ISH

\begin{tabular}{|c|c|c|c|c|c|c|c|}
\hline Number & Gender & Age & Pathological type & Site & SERS & ISH & IHC \\
\hline $\mathrm{PI}$ & Male & 61 & WHO type III & Cervical lymph node & Positive & Positive & Positive \\
\hline P2 & Male & 51 & WHO type III & Cervical lymph node & Positive & Positive & Positive \\
\hline P3 & Male & 45 & WHO type III & Cervical lymph node & Positive & Positive & Positive \\
\hline P4 & Male & 55 & WHO type III & Cervical lymph node & Positive & Positive & Negative \\
\hline P5 & Male & 50 & WHO type III & Cervical lymph node & Positive & Positive & Positive \\
\hline P6 & Male & 52 & WHO type III & Cervical lymph node & Positive & Positive & Positive \\
\hline P7 & Male & 46 & WHO type III & Cervical lymph node & Positive & Positive & Positive \\
\hline P8 & Female & 38 & WHO type III & Cervical lymph node & Negative & Positive & Positive \\
\hline P9 & Female & 32 & WHO type III & Cervical lymph node & Positive & Positive & Positive \\
\hline PIO & Male & 57 & WHO type III & Cervical lymph node & Positive & Positive & Negative \\
\hline PII & Male & 55 & WHO type III & Cervical lymph node & Positive & Positive & Positive \\
\hline $\mathrm{PI} 2$ & Male & 44 & WHO type III & Cervical lymph node & Positive & Positive & Positive \\
\hline $\mathrm{PI} 3$ & Male & 44 & WHO type III & Cervical lymph node & Positive & Positive & Negative \\
\hline $\mathrm{PI} 4$ & Male & 41 & WHO type III & Cervical lymph node & Positive & Positive & Negative \\
\hline PI5 & Male & 57 & WHO type III & Cervical lymph node & Positive & Positive & Positive \\
\hline PI6 & Male & 46 & WHO type III & Cervical lymph node & Positive & Positive & Positive \\
\hline PI7 & Male & 49 & WHO type III & Cervical lymph node & Positive & Positive & Positive \\
\hline PI8 & Male & 62 & WHO type III & Cervical lymph node & Positive & Positive & Positive \\
\hline PIO & Female & 48 & WHO type III & Cervical lymph node & Positive & Positive & Negative \\
\hline P20 & Male & 39 & WHO type III & Cervical lymph node & Positive & Positive & Negative \\
\hline P2I & Male & 56 & WHO type III & Cervical lymph node & Positive & Positive & Positive \\
\hline P22 & Male & 59 & WHO type II & Nasopharynx & Positive & Positive & Negative \\
\hline P23 & Male & 58 & WHO type III & Nasopharynx & Positive & Positive & Positive \\
\hline P24 & Male & 53 & WHO type III & Nasopharynx & Positive & Positive & Positive \\
\hline P25 & Male & 64 & WHO type III & Nasopharynx & Positive & Positive & Negative \\
\hline P26 & Male & 66 & WHO type III & Nasopharynx & Positive & Positive & Positive \\
\hline P27 & Male & 45 & WHO type III & Nasopharynx & Positive & Positive & Negative \\
\hline P28 & Male & 37 & WHO type III & Nasopharynx & Positive & Positive & Negative \\
\hline P29 & Male & 44 & WHO type III & Nasopharynx & Positive & Positive & Positive \\
\hline P30 & Male & 65 & WHO type III & Nasopharynx & Positive & Positive & Positive \\
\hline P3I & Female & 56 & WHO type III & Nasopharynx & Positive & Positive & Negative \\
\hline P32 & Male & 47 & WHO type III & Nasopharynx & Positive & Positive & Negative \\
\hline P33 & Male & 57 & WHO type III & Nasopharynx & Positive & Positive & Positive \\
\hline P34 & Male & 45 & WHO type III & Nasopharynx & Positive & Positive & Positive \\
\hline Average age & \multicolumn{7}{|c|}{$50.7 \pm 8.6$ years } \\
\hline $\begin{array}{l}\text { Gender ratio } \\
\text { (male/female) }\end{array}$ & $30 / 4$ & & & & & & \\
\hline $\begin{array}{l}\text { Healthy subjects } \\
(\mathrm{n}=20)\end{array}$ & $\begin{array}{l}16 \text { male/ } \\
4 \text { female }\end{array}$ & $42.0 \pm 14.9$ & $\begin{array}{l}\text { Normal nasopharyngeal } \\
\text { epithelial tissues }\end{array}$ & $\begin{array}{l}\text { All from } \\
\text { nasopharynx }\end{array}$ & $\begin{array}{l}\text { All are } \\
\text { negative }\end{array}$ & $\begin{array}{l}\text { All are } \\
\text { negative }\end{array}$ & $\begin{array}{l}\text { All are } \\
\text { negative }\end{array}$ \\
\hline
\end{tabular}

Note: WHO type III is non-keratinizing undifferentiated form of NPC; WHO type II is non-keratinizing differentiated form of NPC.

Abbreviations: EBV, Epstein-Barr virus; EBER, EBV encoded small RNA; IHC, immunohistochemistry; ISH, in situ hybridization; LMPI, latent membrane protein I; SERS, surface-enhanced Raman scattering; WHO, World Health Organization.

\section{Publish your work in this journal}

The International Journal of Nanomedicine is an international, peerreviewed journal focusing on the application of nanotechnology in diagnostics, therapeutics, and drug delivery systems throughout the biomedical field. This journal is indexed on PubMed Central, MedLine, CAS, SciSearch ${ }^{\circledR}$, Current Contents ${ }^{\circledR} /$ Clinical Medicine,
Journal Citation Reports/Science Edition, EMBase, Scopus and the Elsevier Bibliographic databases. The manuscript management system is completely online and includes a very quick and fair peer-review system, which is all easy to use. Visit http://www.dovepress.com/ testimonials.php to read real quotes from published authors. 